\title{
Modernización del transporte público en la periferia urbana: ¿el fin del transporte informal? Caso de estudio: sistema de cable aéreo Transmicable en la localidad de Ciudad Bolívar, Bogotá - Colombia
}

Modernização do transporte público na periferia urbana: o fim do transporte informal? Estudo de caso: sistema de cabos aéreos "Transmicable" em Ciudad Bolívar, Bogotá - Colômbia

Public transport modernization in the urban periphery: is the end of informal transport? Case study: overhead cable system "TransMiCable" in Ciudad Bolívar district, Bogotá - Colombia

Jose Ricardo Villar-Uribe[a] (D)

[a] Universidad Piloto de Colombia, Bogotá, Colombia

Cómo citar: Villar-Uribe, J. R. (2021). Modernización del transporte público en la periferia urbana: ¿el fin del transporte informal? Caso de estudio: sistema de cable aéreo Transmicable en la localidad de Ciudad Bolívar, Bogotá - Colombia. urbe. Revista Brasileira de Gestão Urbana, 13, e20190367. https://doi.org/10.1590/2175-3369.013.e20190367

\section{Resumen}

Varias ciudades latinoamericanas han empezado procesos de modernización del transporte público formal en la periferia urbana en donde se incluye la implementación del sistema de cable aéreo. Sin embargo, ¿estos procesos de modernización suponen el fin de servicios informales de transporte que atienden parte de las necesidades de la movilidad cotidiana de los pobladores de asentamientos periféricos? La presente investigación de tipo mixto analiza la incidencia del sistema Transmicable (TMC) de la localidad Ciudad Bolívar (Bogotá, Colombia) en la operación del transporte informal (TI). Los resultados muestran que la incursión del sistema TMC no tuvo mayores afectaciones en el servicio del TI sino que, por el contrario, incentivó la aparición de una nueva ruta que conecta la zona rural de la localidad con el sistema TMC. Adicionalmente, se encontró que el transporte informal permite la realización de un importante número de desplazamientos cotidianos al interior de Ciudad Bolívar que las opciones de transporte público formal no logran servir adecuadamente, lo que da cuenta del proceso de "maduración urbana" que se viene dando en muchos asentamientos populares periféricos latinoamericanos y que requiere un nuevo enfoque en la forma en que se planifica y gestiona el transporte público en la periferia urbana.

Palabras clave: Transporte urbano. Exclusión social. Informalidad.

\section{Resumo}

Várias cidades latino-americanas iniciaram processos de modernização do transporte público formal na periferia urbana, com a implementação do sistema de cabos aéreos. No entanto, esses processos de modernização significam o fim dos serviços de transporte informal que atendem parte das necessidades diárias de mobilidade da população de assentamentos periféricos? A presente pesquisa, de tipo misto, analisa a incidência do sistema Transmicable (TMC) de Ciudad Bolívar (Bogotá, Colômbia) na operação de transporte informal (TI). Os resultados mostram que a incursão do sistema TMC não teve grandes efeitos no serviço de TI e, pelo contrário, incentivou o surgimento de uma nova rota que conecta a área rural da cidade ao sistema TMC. Além disso, verificou-se que o transporte informal permite um número significativo de viagens diárias ao interior de Ciudad Bolívar para as quais as opções formais de transporte

JRVU es arquitecto, master en Planeamiento y Desarrollo Urbano Global, e-mail: jose-villar@unipiloto.edu.co, josevillaruribe@hotmail.com 
público não servem adequadamente, o que explica o processo de "amadurecimento urbano" que está ocorrendo em muitos assentamentos populares periféricos da América Latina, e isso requer uma nova abordagem na maneira como o transporte público é planejado e gerenciado na periferia urbana.

Palavras-chave: Transporte urbano. Exclusão social. Informalidade.

\section{Abstract}

Several Latin American cities have embarked on public transport modernization projects, which include the implementation of aerial cable car systems in densely populated peripheral settlements. However, do these modernization processes mean the end of informal transport services that partially cater peripheral dweller's daily mobility needs? Through a mixed method research, this work analyzed the impact over informal transport services as a result of the TransMiCable (TMC) system implementation in Ciudad Bolívar district in Bogotá, Colombia. The results showed that the incursion of the TMC system did not have major effects on the informal transport (IT) service, and conversely, it encouraged the emergence of a new route that connects the rural area of Ciudad Bolivar with the TMC system. Additionally, it was found that informal transport carries a significant number of daily trips to the interior of Ciudad Bolivar district that formal public transportation modes do not adequately address, which in turn reflects the process of "urban maturation" that is taking place in urban peripheral settlements in Latin America, which demands a new approach in planning and managing public transport in peripheral urban locations.

Keywords: Urban transport. Social exclusion. Informality.

\section{Introducción}

La movilidad en asentamientos periféricos de origen informal se ha convertido en un reto para las ciudades. Es así como, producto de una innovación en relación al transporte público, se implementó la tecnología del cable aéreo como componente de la red de transporte público urbano en Medellín (Colombia) en el año 2004, con el objetivo de facilitar el acceso al sistema metro a un gran número de residentes ubicados en zonas periféricas de montaña densamente pobladas (Metro de Medellín, 2012) para mejorar los desplazamientos cotidianos, y por ende, su calidad de vida. Así mismo, la construcción de esta línea de cable vino acompañada por una serie de intervenciones urbanas que incluyen nuevo espacio público, equipamientos educativos e institucionales y una serie de programas sociales impulsados desde la administración local que se enmarcan dentro de lo que se conoce como "Urbanismo social".

En general, la experiencia de Medellín ha sido bien valorada por un gran número de urbanistas, expertos en transporte, actores políticos, el sector multilateral y la sociedad en general, lo que ha llevado a replicar el modelo en varias ciudades latinoamericanas. Entre los beneficios percibidos por la comunidad en general a partir de la implementación de este tipo de proyectos se destacan la reducción en los tiempos de desplazamiento (Heinrichs \& Bernet, 2014), mejoras en la accesibilidad de los usuarios (Bocarejo et al., 2014a) y un sentido simbólico de visibilidad e inclusión social en el tejido de las ciudades (Leibler \& Brand, 2012).

El entusiasmo despertado por el desarrollo de los cables aéreos en el ámbito latinoamericano ha motivado la realización de varias investigaciones. Estos trabajos abordan temas relacionados a la gestión y desarrollo del proyecto, con particular atención en el papel de la institucionalidad (Acevedo, 2012; Acevedo et al., 2012; Coupé et al., 2013), la participación ciudadana (Daste, 2012) y la gobernanza (Dávila \& Brand, 2012). Otros trabajos exploran las limitaciones de la transferencia y réplica de la experiencia de proyectos tipo cable aéreo (Bocarejo et al., 2014b), los impactos sociales y económicos generados por este tipo de proyectos (Alvarez-Rivadulla \& Bocarejo, 2012a, 2012b; Amorín da Silva, 2012; Brand \& Dávila, 2011, 2013; Coupé, 2012; Coupé \& Cardona, 2013; Derpic, 2018; Milan \& Creutzig, 2017; Naranjo, 2012), los impactos de tipo físico- 
territoriales y ambientales asociados a estas intervenciones urbanas (Dávila \& Daste, 2012; Espósito-Galarce \& Silva, 2016; Gakenheimer, 2012; Goodship, 2015) y la incidencia de la puesta en marcha de proyectos de tipo cable aéreo en la experiencia de viaje en la movilidad cotidiana (Escobar et al., 2015; García \& Orozco, 2011; Garsous et al., 2019; Sarmiento et al., 2013).

Este barrido de la literatura permitió detectar algunos vacíos temáticos por explorar, entre los que se encuentra la incidencia de los sistemas tipo cable en la operación del transporte informal (T.I) que con frecuencia se encuentra en las periferias urbanas. El presente artículo aborda este asunto a partir del análisis de la implementación del sistema de cable aéreo conocido como Transmicable (TMC) en la localidad de Ciudad Bolívar (Figura 1) en Bogotá, Colombia.

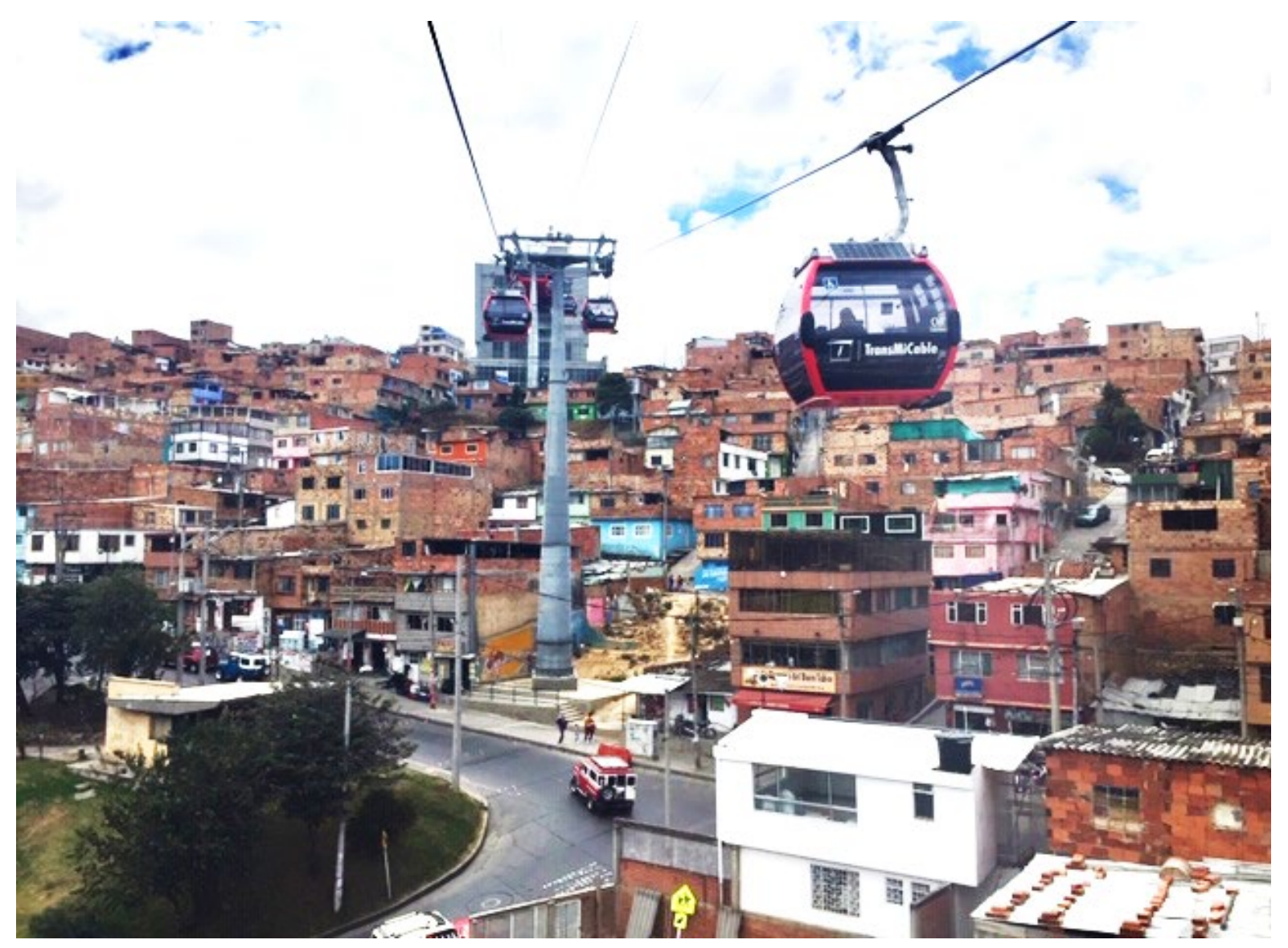

Figura 1 - Vista general del sistema Transmicable en la localidad de Ciudad Bolívar. Fuente: Elaboración propia - Foto tomada por el autor.

\section{El rol del transporte informal en la periferia urbana}

Una de las características de la ciudad latinoamericana atribuíble en gran parte al intenso proceso de urbanización que ha vivido la región, son las amplias extensiones de asentamientos de origen informal localizadas en periferias montañosas (Carrión, 2013). Estos asentamientos que albergan un importante porcentaje de la población que reside en los centros urbanos, históricamente han enfrentado las dificultades que impone una precaria, o en el peor de los casos, inexistente provisión de servicios básicos de consumo colectivo esenciales para el desarrollo humano. Esta ausencia del soporte material necesario para el normal desenvolvimiento de la vida diaria agrava la carga en la cotidianidad de los pobladores de asentamientos periféricos y agudiza las desigualdades en la ciudad, una situación que Kowarick (1996) denominó como "expoliación urbana". De esta manera, quienes habitan en estos asentamientos quedan atrapados en un círculo 
vicioso dominado por la precariedad del habitat y la pobreza (Mitlin \& Satterthwaite, 2013) que sumado a procesos de segregación socio-espacial exacerban su condición vulnerable.

En este contexto, y reconociendo que el acceso a bienes, servicios y oportunidades juega un papel fundamental en el desarrollo de las personas (Litman, 2007) para romper dinámicas que refuerzan la condición de pobreza, las discusiones sobre la producción de espacio urbano que conducen a la emergencia de la ciudad informal cobran relevancia. Al respecto, Pírez (2013) identifica básicamente dos lógicas que rigen esta producción: La primera es la lógica de la necesidad en la que predomina el "autosuministro" y que es de carácter "pre-mercantil" (Jaramillo, 2012, p. 72 apud Piréz, 2013). La segunda se da partir de la producción de bienes y servicios urbanos bajo una lógica "mercantil simple" (Jaramillo, 2012; Abramo, 2012 apud Pírez, 2013) desarrollada por ciertos agentes y que no está motivada por la acumulación de valor (Jaramillo, 2012). Esto último sirve como punto de entrada para discutir sobre el papel del transporte informal en zonas urbanas periféricas, un tema que ha ganado terreno tanto en los debates globales contemporáneos como en la agenda gubernamental de muchas ciudades y que se desarrolla a continuación.

Desde sus inicios los asentamientos periféricos urbanos han tenido que enfrentar un precario servicio de transporte público formal, afectando de esta manera a millones de hogares a nivel global (Jones \& Lucas, 2012). Entre los distintos problemas que aquejan este servicio se resaltan dos en particular que suponen una carga mayor para los pobladores de áreas periféricas y que limitan sus posibilidades de movilidad: 1) una baja cobertura geográfica de la operación del sistema que deja algunos territorios desprovistos de este sevicio, lo que sumado a las condiciones de bajo ingreso y segregación limitan el acceso a la ciudad (Oviedo-Hernández \& Titheridge, 2016); y 2) una reducida disponibilidad de servicios en relación a horarios y frecuencias que afectan negativamente los tiempos de desplazamiento de las personas a lugares de trabajo, comercio, educación, salud, esparcimiento, entre otros (Dimitriou, 2013; Gakenheimer, 1999; Golub et al., 2009; Gwilliam, 2003; Kaltheier, 2002). Como resultado, estas deficiencias socavan el rol de transporte público en el desarrollo de la ciudad (Vasconcellos, 2014) y junto a la falta de alternativas de movilidad conlleva a que la marginalización geográfica agudice fenómenos de exclusión social, lo que contribuye en la aparición de servicios informales de transporte para suplir la demanda existente en estas áreas (Brand \& Dávila, 2011)

La definición del transporte informal aquí utilizada retoma la de Cervero (2000) y Sengers \& Raven (2014) quienes lo definen como un servicio de transporte de pasajeros que carece de permisos y requisitos mínimos para ofrecer su operación. Así mismo, la literatura destaca algunos aspectos importantes que perfilan el rol del transporte informal en la periferia urbana: 1) Frente a una baja cobertura o la total ausencia del servicio de transporte público formal (Cervero, 2000; Gakenheimer, 1999; Vasconcellos, 2014) este tipo de transporte se convierte en el medio por el cual muchos ciudadanos resuelven su movilidad cotidiana, lo que posibilita a estos pobladores tener cierto nivel de acceso a bienes y servicios; 2) el vacío dejado por el transporte público formal resulta en una demanda potencial de pasajeros suficientemente atractiva para propietarios de vehículos privados que posibilita el desarrollo de una actividad laboral y la generación de ingresos (Cervero, 2000; Cervero \& Golub, 2007; Gakenheimer, 1999; Sengers \& Raven, 2014; Shimazaki \& Rahman, 1996) en contextos en el que el desempleo y la pobreza registran altos índices (Clichevsky, 2000; Mitlin \& Satterthwaite, 2013); y 3) teniendo presente el proceso de "maduración urbana" que se viene dando en áreas periféricas populares expresado en una consolidación de lo construído, una ampliación de la oferta de empleo y servicios mediante la construcción de nuevos equipamientos, entre otras (Dureau \& Gouëset, 2011; Lavadinho, 2014) y que se traduce en una mixtura de usos que propicia cierto nivel de proximidad urbana (MirallesGuasch \& Sardà, 2013), el servicio que presta el transporte informal al interior de estos asentamientos permite a los pobladores realizar intercambios sociales y económicos en su cotidianidad, haciendo una contribución importante en el funcionamiento del territorio. 
Lo anterior ha desencadenado toda una serie de discusiones en torno a la movilidad en los bordes periféricos urbanos en donde se pueden observar distintas posturas. Una de ellas apunta a la modernización del transporte público formal, que junto a la implementación de restricciones drásticas por parte de las autoridades busca cerrarle el campo de maniobra al transporte informal (Cervero, 2000; Golub et al., 2009; Vasconcellos; 2014) bajo el argumento que una actividad sin registro ni autorización está limitada para prestar el servicio en condiciones mínimas de seguridad y responsabilidad. Por el contrario, otra postura reconoce el papel del transporte informal para cubrir vacíos en la cobertura de transporte y sugieren la libre circulación de manera que "complemente" la oferta de transporte urbano existente (Cervero, 2000; Cervero \& Golub, 2007; Golub et al., 2009; Lomme, 2008). Una tercera propone la posibilidad de integrar física y tarifariamente el transporte informal con el formal para impulsar un proceso de renovación de flota del transporte informal (Cervero, 2000) y consolidar una red de transporte multimodal (Lomme, 2008). Sin embargo, la decisión sobre cómo abordar el transporte informal dista de ser sencilla, y por el contrario, existen toda una serie de factores sociales, económicos y culturales que hacen aún más compleja la tarea (Villar-Uribe, 2017).

En este artículo la discusión gira en torno al proceso de modernización de transporte en la periferia urbana a partir del análisis de la implementación del sistema de cable aéreo Transmicable (TMC) y su incidencia en la operación del transporte informal. La siguiente sección presenta un panorama general del caso de estudio, con particular énfasis en las condiciones sociales del sector y en el que se habla del proceso de modernización del transporte público formal en la zona de estudio. Seguidamente, se expone la metodología implementada en el desarrollo de este trabajo y en las siguientes secciones se presentan los resultados del trabajo de campo para finalmente exponer las conclusiones.

\section{Contextualización del caso de estudio}

La localidad de Ciudad Bolívar se encuentra ubicada en el suroccidente de Bogotá (Figura 2). Es una localidad con una topografía mayoritariamente montañosa ( $90 \%$ de la localidad) y con un alto componente de área rural (72\%) (Bogotá, 2019). Según la encuesta multipropósito 2017 (Bogotá, 2017a) esta localidad alberga el 16,7\% de la población rural de Bogotá, siendo la tercera localidad con mas habitantes rurales. Así mismo, es la segunda con el mayor índice de pobreza multidimensional $(8,7 \%)$ con una tasa de desempleo del $12,56 \%$, superior a la registrada en Bogotá (10,3\%) (Bogotá, 2017b) y es una localidad que enfrenta similares condiciones de segregación socioespacial registradas en territorios vecinos (Ver Moreno Luna \& Rubiano Bríñez, 2014).

La zona de estudio no ha sido ajena al proceso de modernización del transporte público formal que se ha dado en Bogotá. Este proceso incluye la implementación del sistema de buses de tránsito rápido (BRT) Transmilenio (TM), la puesta en marcha del Sistema Integrado de Transporte Público (SITP) y la construcción del sistema de cable aéreo Transmicable (TMC), y en todos los casos la operación del sistema está a cargo de empresas privadas. La oferta de transporte público formal en la localidad incluye rutas que conectan con diversos lugares de la ciudad gracias a la cobertura del SITP y la operación de buses alimentadores que enlazan la localidad con la estación "Portal Tunal", la cual funciona como terminal de integración con el sistema de buses Transmilenio (Figura 3). Adicionalmente, a finales de diciembre de 2018 se dio apertura a la operación del sistema de cable aéreo TMC en la localidad Ciudad Bolívar el cual cuenta con 3 estaciones en los barrios Juan Pablo II, Manitas, Mirador y Paraíso (Figura 4) que funciona como otro ramal alimentador y facilita la conexión con el sistema Transmilenio en un intercambio modal que se da igualmente en la estación "Portal Tunal". 


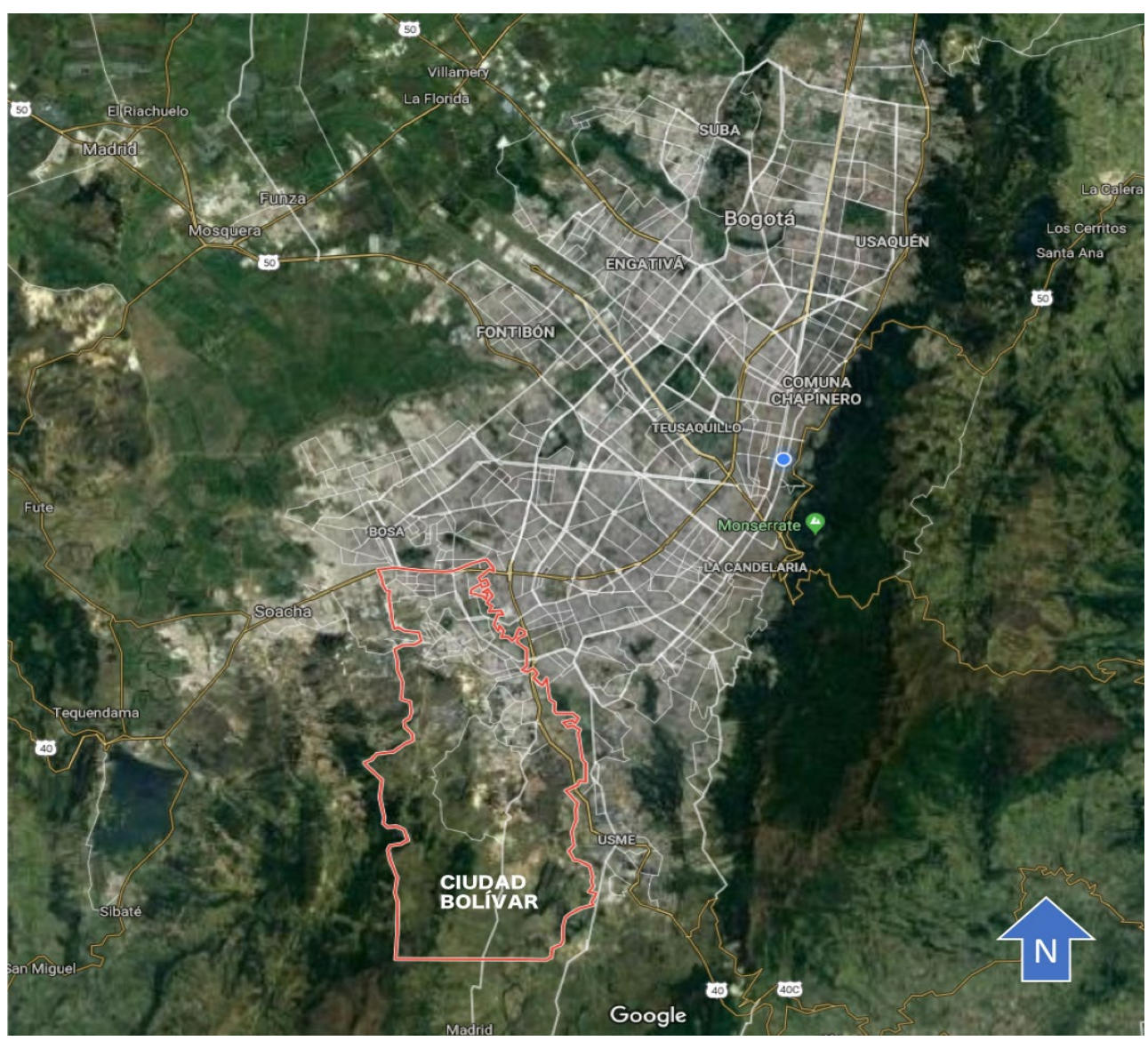

Figura 2 - Ubicación de la localidad Ciudad Bolívar en Bogotá. Adaptado de Google Earth.

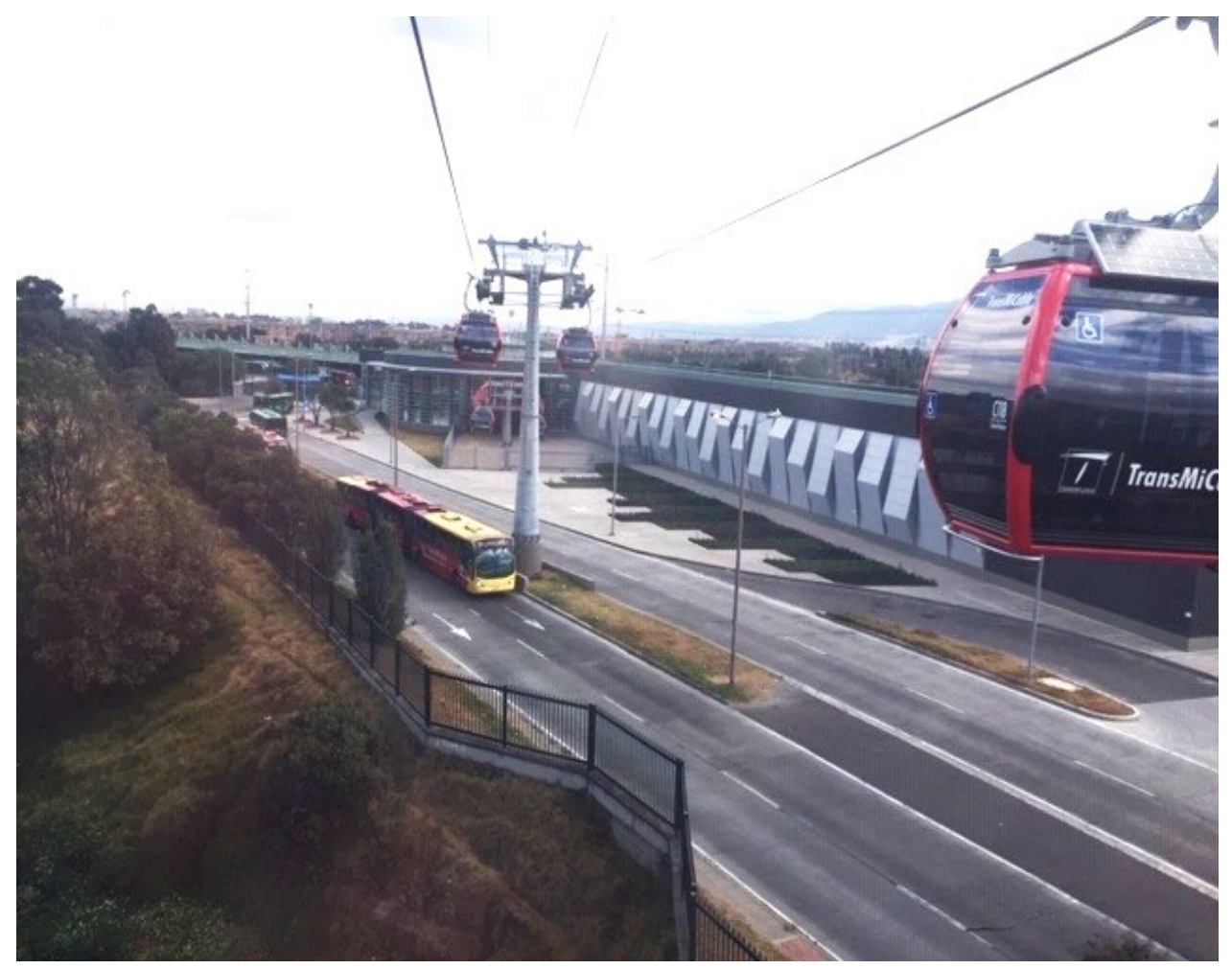

Figura 3 - Vista desde Transmicable hacia estación de intercambio modal "Portal Tunal". Fuente: Elaboración propia - Foto tomada por el autor. 


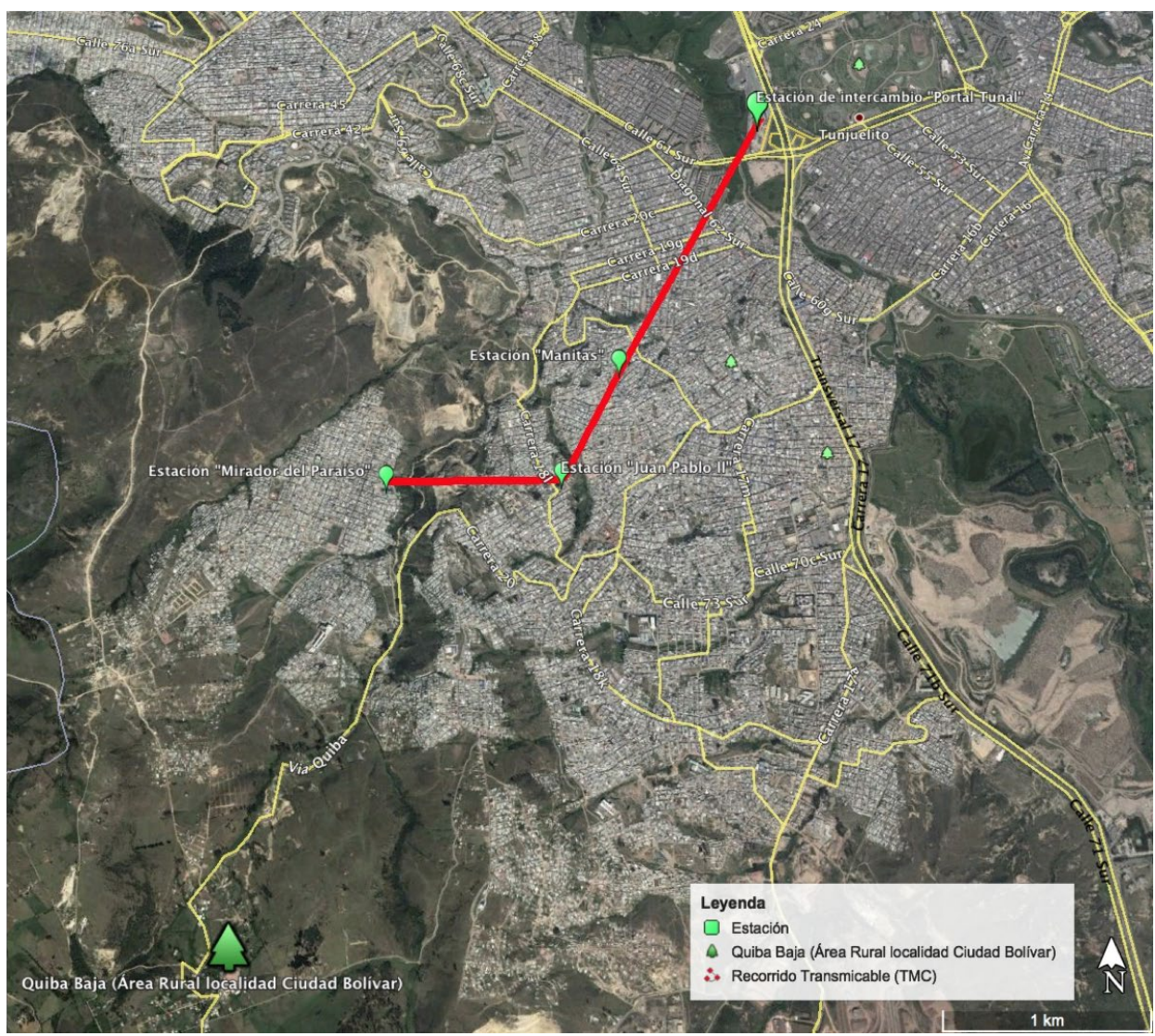

Figura 4 - Mapa del recorrido del sistema Transmicable en Ciudad Bolívar. Adaptado de Google Earth.

A pesar de la existencia de rutas de transporte público, cabe mencionar que la infraestructura viaria presenta muchos desarrollos incompletos que, sumado a las condiciones topográficas de la localidad, dificultan la movilidad de peatones, ciclistas y del tráfico motorizado incluyendo el transporte público (Figura 5). Esta situación no es menor, ya que, además de las limitantes que imponen las periferias urbanas para el desplazamiento peatonal (Ureta, 2008) estos desplazamientos se tornan riesgosos para los habitantes y por lo tanto un gran número de ellos recurren al transporte informal para la realización de viajes de cortas distancias. El presente trabajo se concentró en el caso de la última estación del sistema TMC llamada "Mirador del Paraíso" y su incidencia en el servicio de transporte informal del área de influencia.

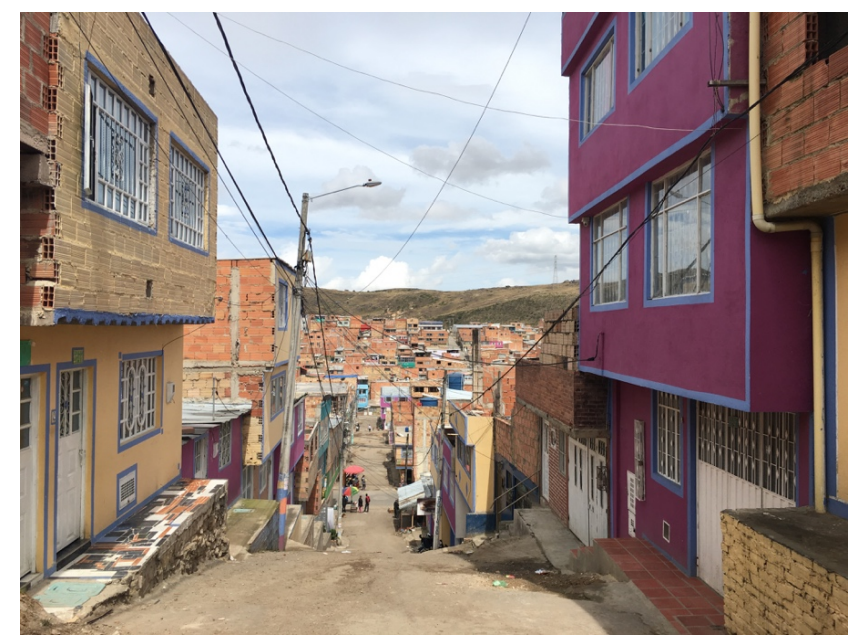

Figura 5 - Infraestructura vial en el barrio El Paraíso. Fuente: Elaboración propia - Foto tomada por el autor. 


\section{Estación "Mirador del Paraíso"}

Esta estación se ubica en la parte de alta de la localidad Ciudad Bolívar, específicamente en los barrios Mirador y Paraíso, siendo la última estación del sistema (Figura 6). El recorrido desde esta estación hacia la estación de intercambio "Portal Tunal" puede tomar alrededor de 15 minutos, lo que representa una reducción de tiempo significativa, ya que según relatos de usuarios realizar esa conexión en bus alimentador podía tomar entre 40 minutos y 1 hora, dependiendo del tráfico vehicular de la zona (Martínez, 2019) .

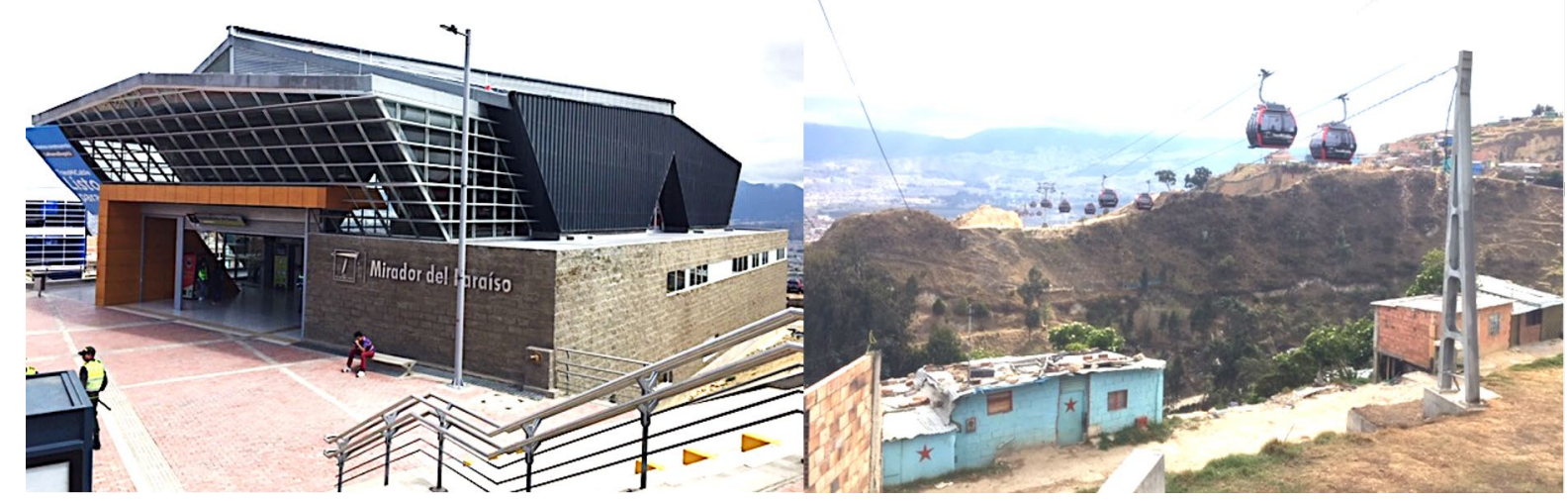

Figuras 6 - Estación "Mirador del Paraíso" y ámbito general donde se ubica la estación. Fuente: Elaboración propia a partir de material fotográfico del autor.

Esta estación es la más próxima a la zona rural de Ciudad Bolívar, donde se encuentra la Vereda Quiba Baja (Figura 7), la cual ha visto un mayor número de visitantes a partir de la entrada en operación del TMC. Así mismo, es pertinente mencionar que el SITP tiene una ruta de transporte público que llega hasta esta vereda.

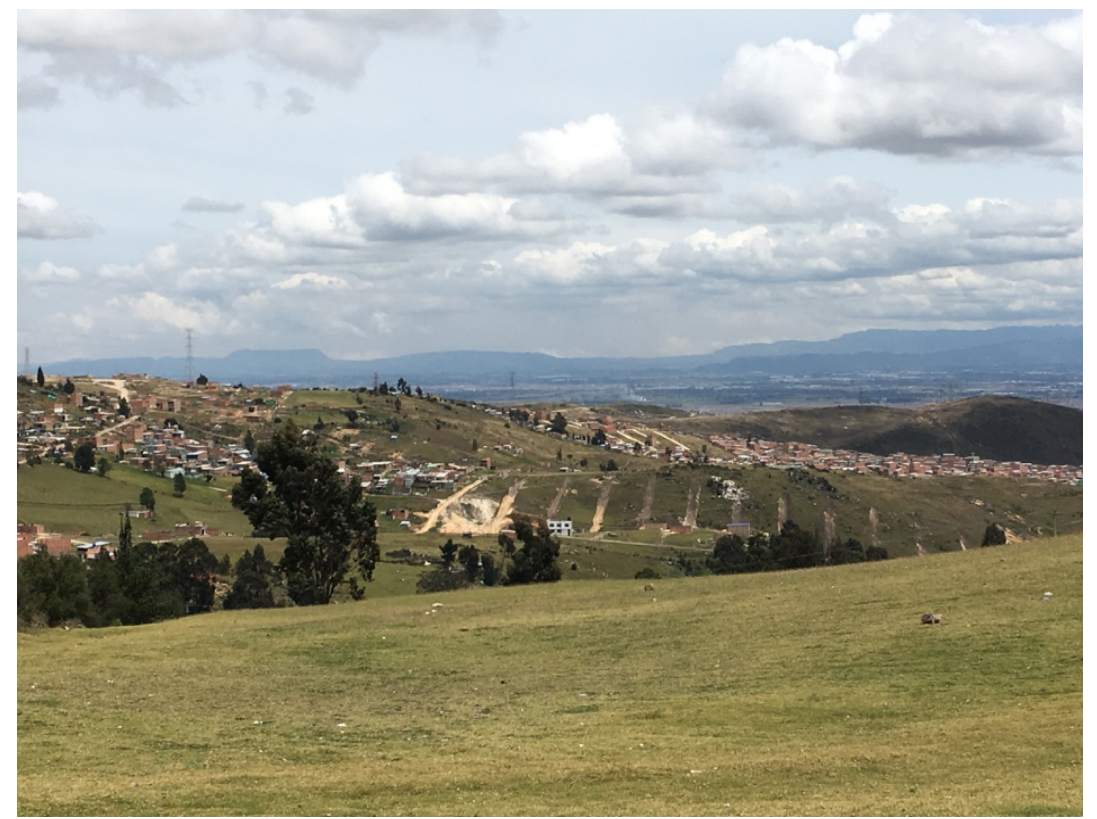

Figura 7 - Vista desde la Vereda Quiba Baja (Área rural de la localidad Ciudad Bolívar). Fuente: Elaboración propia - Foto tomada por el autor. 
Considerando que los cables aéreos conectan una estación con otra y también generan intercambios entre diversas formas de movilidad ya que los usuarios continúan más allá de la estación, esta continuación del viaje es algo que requiere ser entendido y gestionado (Goodship, 2015). En ese sentido, la ubicación de la última estación del sistema TMC "Mirador del Paraíso" despertó el interés por analizar la incidencia de este proyecto en la operación del sistema informal, ya que estos sectores son propicios para la emergencia de este tipo de servicios como respuesta a las deficiencias registradas en la operación del sistema de transporte público formal (Cervero, 2000).

\section{Metodología}

El presente trabajo se enmarca dentro del proyecto de investigación "Movilidad urbana y el entorno como generadores de ciudadanía - Fase 1" realizado por docentes de la Universidad Piloto de Colombia, el cual recurre al estudio de caso como estrategia metodológica para comprender fenómenos urbanos en contextos singulares (Páramo, 2011). Este artículo describe y analiza el comportamiento del transporte informal frente a la incursión del TMC como parte del proceso de modernización en la oferta de transporte público en la periferia urbana. En particular, se indaga hasta qué punto la implementación de nuevos corredores de transporte público formal logran sustituir el servicio que ofrece el transporte informal. El caso estudiado corresponde al área de influencia de la última estación del sistema TMC "Mirador del Paraíso" dadas las características contextuales expuestas en la sección anterior.

La estrategia metodológica de tipo mixto incluye la revisión documental, explotación de fuentes secundarias, realización de encuestas, ejercicios de observación no participante, breves conversaciones con actores involucrados en la operación del transporte informal y viajes comentados. La recolección de datos se hizo en dos momentos. Primero se realizó una encuesta general dirigida específicamente a usuarios del sistema TMC que cubrió diferentes aspectos del proyecto de investigación macro, entre los que se preguntaba sobre el uso del transporte informal. En este caso se realizaron 239 encuestas en un universo de 21.000 usuarios en día hábil (Transmilenio, 2019). En relación al uso del transporte informal los resultados logrados se compararon con los datos que arrojó la encuesta realizada por el Instituto de Desarrollo Urbano (IDU) a 301 personas entre residentes y comerciantes de la Unidad de Planeamiento Zonal 67 - Lucero ${ }^{1}$, la cual cuenta con un margen de error del 6\% y una confiabilidad del 95\% (IDU, 2019)

Teniendo presente que la especificidad de la población encuestada podría sesgar los resultados logrados, seguidamente se realizó un ejercicio de observación no participante que sirvió para identificar la dinámica del transporte informal en la zona de estudio y algunas de sus características, lo cual permitió adelantar un segundo momento en el trabajo de campo. Este segundo momento se enfocó en reconocer algunos aspectos del transporte informal y consistió en la realización de encuestas a conductores y usuarios en dos rutas establecidas en el área de estudio para tener un mejor conocimiento en cuanto a la operación, organización, cobertura del servicio, tarifas, entre otros. Este ejercicio permitió establecer que el tamaño del grupo de operadores en cada ruta es de pequeña escala. Mientras que en la ruta "Paraíso San Francisco" el número de operadores varía entre 21 y 24, en la ruta "Estación TMC "Mirador del Paraíso" - Quiba Baja" la variación en el número de operadores es entre 18 y 20 . Teniendo presente que la totalidad de la flota en ambas rutas está compuesta por vehículos particulares, la operación está condicionada por la restricción a la circulación de vehículos de este tipo conocida como "pico y placa" 2 y que se aplica en la ciudad de Bogotá. Esta restricción lleva a que la operación del transporte informal en

\footnotetext{
${ }^{1}$ La UPZ 67 Lucero es una subdivisión territorial al interior de la Localidad Ciudad Bolívar, en donde se ubican las estaciones del sistema Transmicable y su área de influencia. Cuenta con una población de 196.262 habitantes (Veeduría Distrital, 2019).

2 La medida del "Pico y Placa" es una restricción a la circulación de vehículos que se da de lunes a viernes en horarios de alta congestión (6:00 a. m. a 8:30 a. m. y de 3:00 p. m. a 7:30 p. m.). La forma en que se aplica esta restricción depende del último número de la matricula del vehículo. Así, los vehículos cuyo último dígito sea un número impar, no podrán circular los días hábiles impares del mes (Por ejemplo, si el último dígito de un vehículo es 5 entonces no podrá circular en los días impares del mes). Lo mismo sucede con las matrículas que terminan en número par, que no podrán circular en los días hábiles con fecha par.
} 
días hábiles en ambas rutas se realice con el 50\% de la flota disponible en promedio. Es decir, al día pueden estar operando entre 10 y 13 vehículos en cada ruta.

En cuanto al número de encuestas a operadores del transporte informal se logró realizar un total de 11, es decir cerca del 50\% de los conductores disponibles en el día en que se realizó la actividad, que se dividieron así: 6 encuestas (55\%) en la ruta "Paraíso - San Francisco" y 5 encuestas (45\%) en la ruta "Estación TMC "Mirador del Paraíso" - Quiba Baja”.

En relación a los usuarios se realizaron 22 encuestas a personas mayores de edad en las dos rutas mencionadas de las cuales el $45 \%$ son mujeres y el restante $55 \%$ hombres. Adicionalmente, se realizaron breves conversaciones con dos despachadores (uno por ruta) y conversaciones casuales con usuarios a lo largo de cuatro viajes (dos por ruta) para conocer aspectos adicionales de la operación del transporte informal. Las encuestas se realizaron en un día hábil de la semana de manera aleatoria, anónima y voluntaria. Se considera que la muestra lograda ofrece unos resultados que permiten identificar algunas características y dinámicas claves del funcionamiento del transporte informal para realizar un análisis y discusión de la situación estudiada.

\section{Resultados}

A primera vista, el uso del transporte informal en la movilidad cotidiana de la personas en la zona de estudio pareciera ser marginal. Según la encuesta realizada por el Instituto de Desarrollo Urbano (IDU, 2019) solo el $4 \%$ utiliza este medio con mucha frecuencia y un $10 \%$ lo utiliza muy poco. Por su parte, la encuesta realizada a usuarios del sistema Transmicable en el marco de este proyecto de investigación arrojó que solo un 1\% utiliza el transporte informal para llegar al sistema TMC en sus viajes de ida (Trabajo, educación, diligencias, ocio, etc.). En cuanto a los viajes de regreso esta cifra sube al 4\%, incremento que se da principalmente por horarios de trabajo de los usuarios que superan el de la operación del sistema TMC.

Sin embargo, el principal hallazgo que se deriva de los resultados logrados es que la operación del transporte informal (T.I) no se ha visto diezmada en la zona de estudio a pesar de la entrada en operación del sistema TMC y que hace parte del proceso de modernización del transporte público en la localidad Ciudad Bolívar. A pesar que la mayoría de los usuarios del transporte informal dicen tener acceso desde sus hogares a una o varias modalidades del transporte público formal que operan en la zona (Transmicable, buses alimentadores del sistema Transmilenio (TM), SITP, SITP provisional) (Figura 8), mediante el ejercicio de indagación se encontró una importante presencia del transporte informal en la zona de estudio, y se comprobó la aparición de una nueva ruta que opera informalmente conectando la última estación del sistema TMC con la zona rural de la localidad. Lo anterior fue ratificado por el 82\% de los operadores de transporte informal encuestados, quienes manifestaron que han visto la aparición de nuevas rutas (Figura 9)

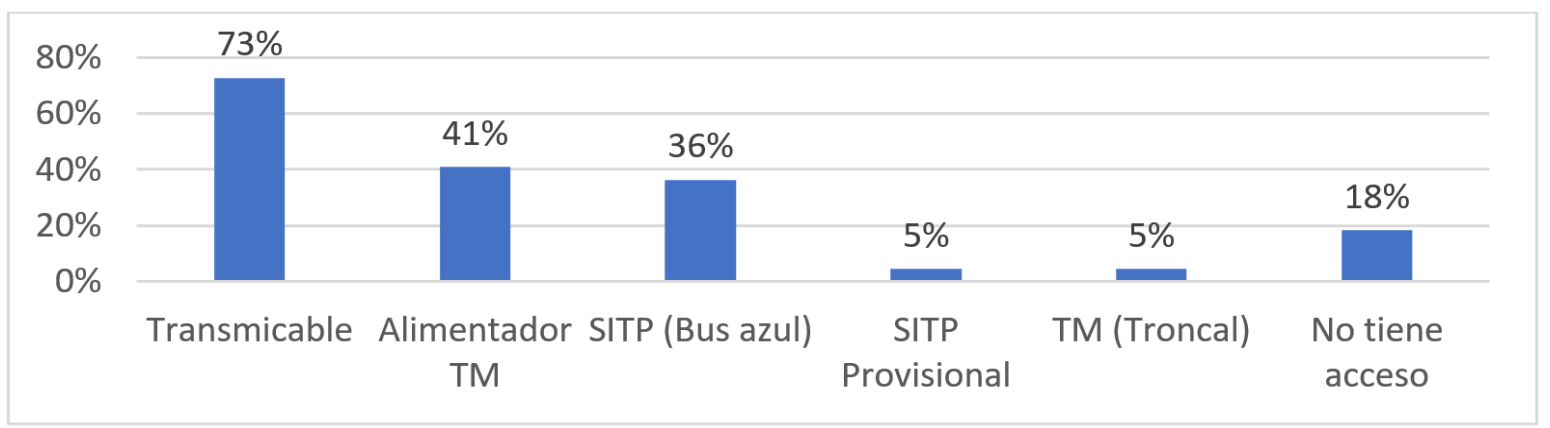

Figura 8 - Porcentaje de usuarios del transporte informal encuestados que dicen tener acceso al transporte público formal en la zona de estudio en sus distintas modalidades: Transmicable, servicio Alimentador del sistema Transmilenio (TM), Sistema Integrado de Transporte Público (SITP), servicio Troncal del sistema Transmilenio (TM). Fuente: Elaboración propia a partir de encuesta del proyecto de investigación. 


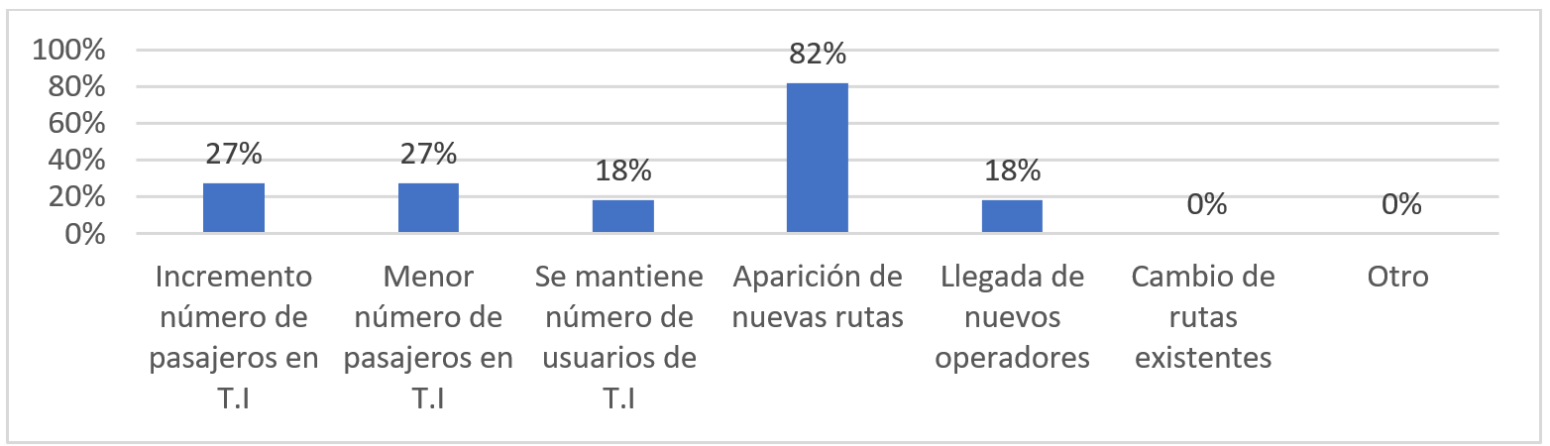

Figura 9 - Percepción de los operadores del transporte informal en los cambios en la operación del servicio con la entrada en operación del sistema Transmicable (TMC). Fuente: Elaboración propia a partir de encuesta del proyecto de investigación.

$\mathrm{Al}$ indagar entre usuarios del transporte informal sobre las razones para no utilizar las opciones disponibles del transporte público formal, el $32 \%$ señaló que el tiempo de viaje es mayor, mientras que un $9 \%$ manifestó que los tiempos de espera eran muy largos. Ambos resultados suman un $41 \%$ de las respuestas, lo cual expresa la valoración que los usuarios hacen del tiempo en sus desplazamientos diarios. Mientras que un vehículo de transporte informal es despachado cada 5 a 7 minutos en promedio, según relatos de varios usuarios la espera de la ruta del bus del SITP o del servicio alimentador de Transmilenio puede tomar más de 30 minutos. Por otro lado, el 14\% comentó que las opciones de transporte público formal disponibles no permiten conectar con los destinos a los que se dirigen normalmente, lo que deja entrever la discrepancia entre el planeamiento y gestión del transporte público frente a las realidades de este territorio periférico (Ver Figura 10).

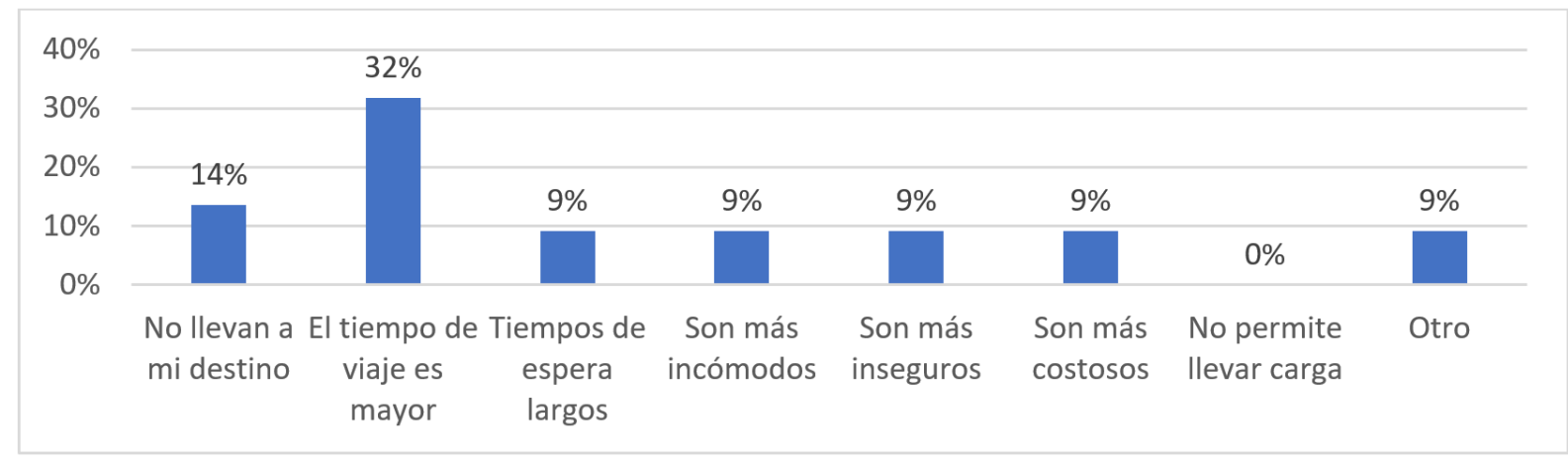

Figura 10 - Razones por las cuales los encuestados no utilizan las opciones de transporte público formal en la zona de estudio. Fuente: Elaboración propia a partir de encuesta del proyecto de investigación.

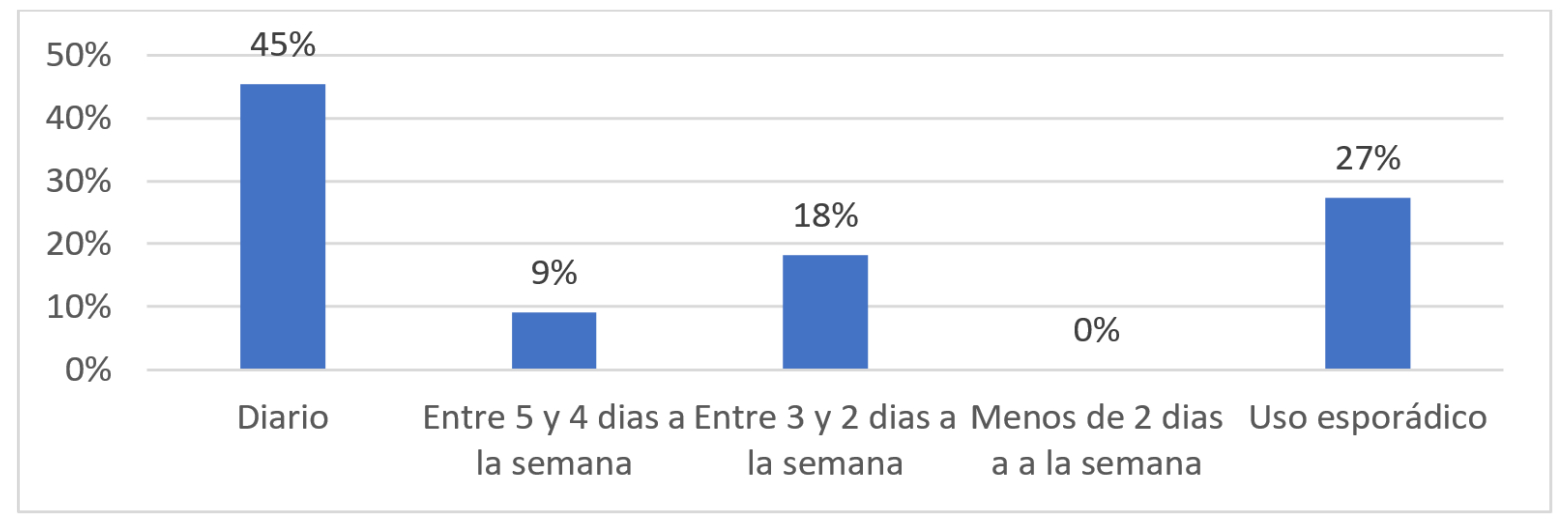

Figura 11 - Frecuencia en el uso del transporte informal. Fuente: Elaboración propia a partir de encuesta del proyecto de investigación. 
Así mismo, la frecuencia en el uso del transporte informal por parte de los usuarios encuestados es alta, ya que un 54\% manifiesta usarlo diariamente (45\%) o entre 4 y 5 días a la semana (9\%) (Figura 11). Igualmente, el servicio goza de altos niveles de satisfacción entre los usuarios en donde el $96 \%$ de los encuestados expresa una alta satisfacción ("totalmente satisfecho" 64\%, o "muy satisfecho" 32\%) (Ver Figura 12). Entre las razones que motivan el uso del transporte informal se destacan aquellas en relación a la comodidad (50\%), a la disponibilidad del servicio (55\%) y a la conveniencia según las necesidades de desplazamiento (50\%) (Ver Figura 13).

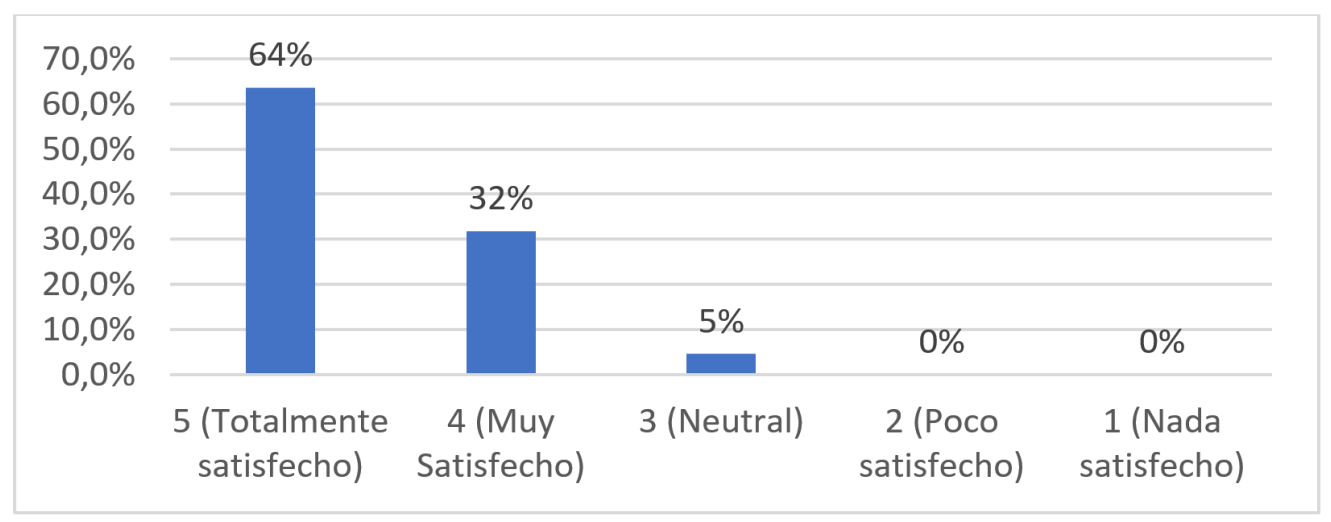

Figura 12 - Nivel de satisfacción del servicio ofrecido por el transporte informal según los usuarios encuestados. Fuente: Elaboración propia a partir de encuesta del proyecto de investigación.

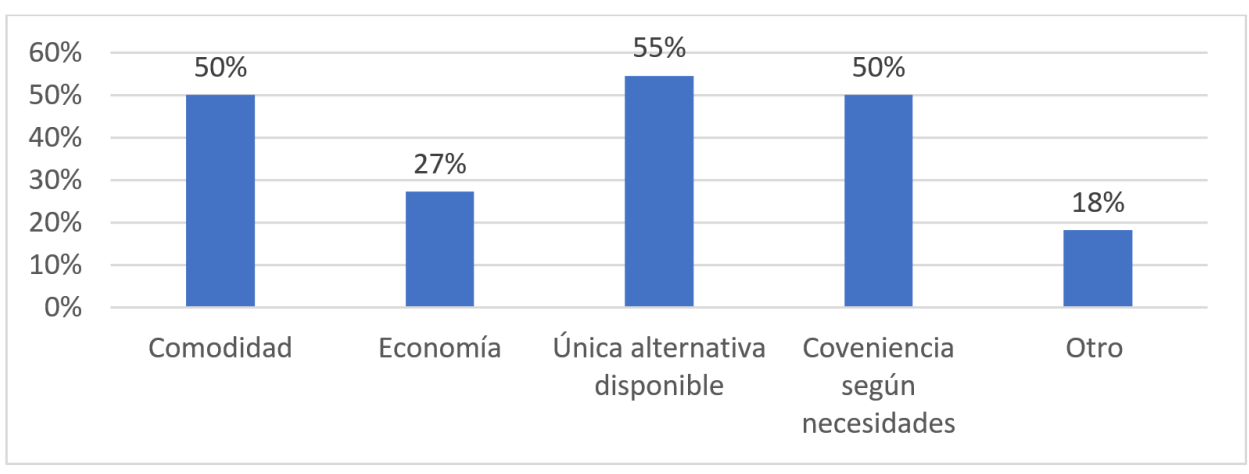

Figura 13 - Razones de los usuarios que motivan el uso del transporte informal. Fuente: Elaboración propia a partir de encuesta del proyecto de investigación.

Otro dato de gran relevancia y que se vincula con lo mencionado en el párrafo anterior, es el carácter local que tiene la operación del transporte informal. Mientras el 95\% de los usuarios viven en la localidad de Ciudad Bolivar (68\% en zona úrbana y $27 \%$ en área rural) (Figura 14), el 68\% de los usuarios encuestados afirma que el lugar de destino final de sus viajes se localiza dentro de la localidad (59\% en la parte urbana y $9 \%$ en la zona rural) (Figura 15). Entre los usuarios que se desplazan al interior de la localidad (es decir que viven y se movilizan en Ciudad Bolívar), el 64\% lo hace por motivos de trabajo. A la luz de estos resultados se puede decir que el servicio ofrecido por el transporte informal entiende y se adapta a las realidades sociales y económicas que se vienen dando en la localidad de de Ciudad Bolívar. Por su parte, el $32 \%$ de los usuarios del T.I encuestados indican que el destino de sus viajes cotidianos se ubica lejos de la localidad (Figura 15), principalmente en áreas a las que se puede llegar en el sistema Transmilenio o SITP, para lo cual realizan el intercambio modal entre transporte informal y el sistema TMC, lo que testifica la dinámica de "complementariedad" que se puede encontrar entre el servicio formal e informal en algunas rutinas de viaje de los pobladores de áreas periféricas 


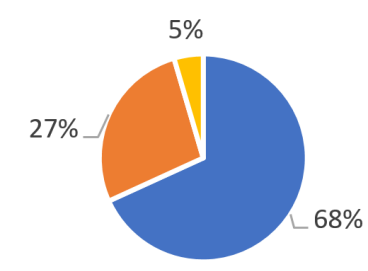

\footnotetext{
- En la localidad Ciudad Bolívar (Área Urbana)

- En la localidad Ciudad Bolívar (Área rural)

- Fuera de la localidad y lejos de la zona de operación
}

Figura 14 - Lugar de residencial del usuario del transporte informal. Fuente: Elaboración propia a partir de encuesta del proyecto de investigación.

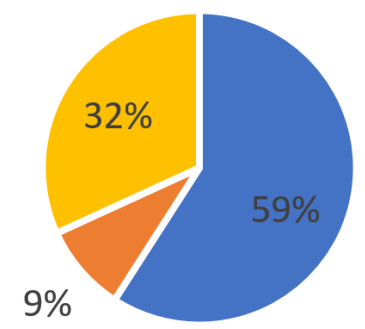

$$
\begin{aligned}
& \text { - En la localidad Ciudad Bolivar (Área Urbana) } \\
& \text { - En la localidad Ciudad Bolivar (Área rural) } \\
& \text { - Fuera de la localidad y lejos de la zona de operación }
\end{aligned}
$$

Figura 15 - Lugar de destino de los viajes en transporte informal. Fuente: Elaboración propia a partir de encuesta del proyecto de investigación.

\section{Discusión y conclusiones}

$\mathrm{Al}$ analizar en conjunto los resultados en relación a la operación del transporte informal (T.I) en la zona de influencia de la estación "Mirador del Paraíso", se puede concluir que la llegada del sistema TMC como parte del proceso de modernización del transporte público en Ciudad Bolívar no ha significado la desaparición o reducción en la oferta de servicios informales de transporte. Por el contrario, se ha visto que este transporte se ha expandido en la zona de estudio con el establecimiento de una nueva ruta denominada "Estación TMC Mirador del Paraíso - Quiba Baja" que conecta el sistema TMC con la zona rural de la localidad, lo que permite ampliar el acceso al sistema TMC. Por su parte, en rutas previamente establecidas como la de "Paraíso - San Francisco" la operación sigue estable sin mayores repercusiones. De hecho, en algunas situaciones especiales los operadores de esta ruta entran a apoyar el servicio de transporte desde la estación del TMC a la zona rural de la localidad cuando la demanda desborda la capacidad del naciente grupo de operadores que proveen el servicio en la ruta "Estación TMC Mirador del Paraíso - Quiba Baja".

La continuidad de la prestación del servicio del transporte informal y la emergencia de una nueva ruta en la zona de estudio pueden entenderse a partir del entendimiento y adaptación que pareciera tener este tipo de transporte frente a los procesos de "maduración urbana" (Dureau \& Gouëset, 2011) que se vienen dando en las zonas periféricas urbanas en ciudades latinoamericanas, que incluye una ampliación en la oferta de empleo y de servicios, forjando la aparición de una mixtura de usos que propicia ciertas condiciones de proximidad urbana (Miralles-Guasch \& Sardà, 2013). En el caso estudiado, esto se ve 
reflejado en el número de usuarios que residen y trabajan en la localidad y que utilizan el T.I, a pesar de la presencia de distintas modalidades de transporte público formal a las que el $82 \%$ de los encuestados dice tener acceso.

Lo anterior supone una fuerte confrontación a la manera en que se planea y gestiona el transporte público formal en áreas periféricas urbanas. Si bien en el caso de Bogotá predominan los desplazamientos hacia zonas de alta concentración de empleo y oferta educativa como son el área central, la zona norte y la zona occidente de la ciudad a las que se puede llegar mediante el sistema BRT Transmilenio o el SITP, los resultados arrojados dejan entrever que también existe una dinámica de intercambios sociales y comerciales de tipo endógeno en la localidad Ciudad Bolívar que no son adecuadamente atendidas por el transporte público formal. Una de las razones que podría explicar esta deficiencia de este servicio es el hecho que la operación del sistema está en manos de grandes empresas privadas, siguiendo el modelo neoliberal que se ha venido instalando en la región en la provisión de servicios urbanos. Esto conduce a que el servicio está diseñado para ser rentable bajo una lógica de oferta y demanda, y por lo tanto, está orientado en atender desplazamientos hacia zonas de alta demanda. Por el contrario, a pesar de la existencia de cobertura del servicio en lugares de "baja demanda", la baja frecuencia en el despacho de flota conlleva a largos tiempos de espera que afectan a los pobladores en áreas periféricas, tal y como sucede en área de estudio. Lo anterior abre el espacio para la aparición y consolidación de servicios informales de transporte que entienden la dinámica de movilidad del territorio, se adaptan a las condiciones de lugar, desarrollan un esquema de servicio bajo una lógica "mercantíl-simple" (Jaramillo, 2012) que posibilita la generación de ingresos (aunque en muchos casos ni siquiera iguale el salario mínimo legal vigente) a un remanente de mano de obra desempleada residente en la zona, y que en últimas, contribuyen en el funcionamiento del territorio al permitir toda una serie de intercambios sociales y económicos que vitalizan la localidad.

Frente a este escenario, vale la pena preguntarse entonces ¿hasta qué punto la operación del transporte informal se constituye en una solución viable y adecuada para los retos que impone la movilidad en las periferias urbanas?. La respuesta está lejos de romantizar la idea del transporte informal como la solución a los problemas de movilidad en la periferia urbana, y por el contrario reconoce el conjunto de impactos negativos en el territorio y la sociedad en general que conlleva un servicio sin registro, control ni regulación (Cervero, 2000), y que para el caso de comunidades asentadas en áreas periféricas urbanas podrían agudizar su vulnerabilidad.

Sin embargo, debe llamarse la atención sobre la posibilidad de diseñar otros esquemas de regulación y organización que complementen el conjunto de instrumentos para la gestión institucional del transporte urbano, que permitan abordar la vasta complejidad implicita en el entramado de las distintas realidades sociales presentes en las urbes latinoamericanas. El caso de Ciudad Bolívar evidencia que, así como se viene dando un proceso de maduración territorial, también existen iniciativas locales que funcionan bajo una estructura organizacional sencilla que dan respuesta al problema de la movilidad cotidiana de un importante número de pobladores en áreas periféricas y que han probado su capacidad de adaptación y resiliencia frente al proceso de modernización del transporte público que se viene dando en el lugar, dejando algunas lecciones valiosas para abordar el reto que impone la movilidad en periferia.

Estas lecciones podrían capitalizarse en un proceso de organización del transporte bajo un esquema participativo que propicie condiciones realistas para los participantes (actuales actores involucrados en la operación del transporte informal), que permita la evolución del servicio, es decir, tanto el mejoramiento de las condiciones para los usuarios como de las condiciones laborales para los operadores, y el fortalecimiento de un sector de la economía local que contribuya en la generación de empleo mediante la consolidación de un ecosistema microempresarial de escala local que gire en torno a la prestación del servicio de transporte público en un territorio con una alta tasa de desempleo e informalidad. En el caso colombiano, esta transformación puede apalancarse en lo dispuesto en el artículo 74을 del Plan Nacional de Desarrollo 2018-2022 dando continuidad a lo consignado en la Ley 1753 de 2015, que establece otras fuentes de financiación como los fondos de estabilización, el pago de estacionamiento público, los cargos por congestión, entre otras, con el objetivo de lograr la sostenibilidad 
de la operación del transporte público, lo que podría significar un cambio en el enfoque bajo el cual se hace el planeamiento, gestión y operación de un servicio urbano esencial.

En este sentido, futuros trabajos podrían profundizar sobre diversos aspectos del transporte informal tales como su estructura organizacional, el esquema operacional, el perfil socioeconómico de quienes están vinculados en la operación del servicio, las posbilidades de mutación del servicio hacia sencillos esquemas de formalización, entre otros, para alimentar la discusión frente a la necesidad de desarrollar un abordaje alternativo a los retos que suponen la movilidad cotidiana en la periferia urbana.

\section{Referencias}

Abramo, P. (2012). La ciudad informal com-fusa: el mercado y la producción de la territorialidad urbana popular. In C. E. Salazar (Ed.), Irregular: suelo y mercado en América Latina (pp. 85-124). México: El Colegio de México.

Acevedo, J. (2012). Los cables aéreos en zonas marginales urbanas: un desafío institucional. Bitácora Urbano Territorial, 2(21).

Acevedo, J., Velásquez, J. M., \& Bocarejo, J. P. (2012). La promesa presidencial de un cable aéreo: Limitaciones institucionales y realidades políticas. In J. D. Dávila (Ed.), Movilidad urbana y pobreza: aprendizajes de Medellín y Soacha, Colombia (pp. 130-136). Londres: Development Planning Unit, UCL, y Universidad Nacional de Colombia.

Alvarez-Rivadulla, M. J., \& Bocarejo, D. (2012a). Contexto socio-económico de la comuna 4 de Soacha: vulnerabilidad, encierro, estigma. In J. D. Dávila (Ed.), Movilidad urbana y pobreza: aprendizajes de Medellín y Soacha, Colombia (pp. 137-142). Londres: Development Planning Unit, UCL, y Universidad Nacional de Colombia.

Alvarez-Rivadulla, M. J., \& Bocarejo, D. (2012b). La esperanza de ser vistos: percepciones de los habitantes de la Comuna 4 frente a la posible construcción de un cable aéreo. In J. D. Dávila (Ed.), Movilidad urbana y pobreza: aprendizajes de Medellín y Soacha, Colombia (pp. 143-148). Londres: Development Planning Unit, UCL, y Universidad Nacional de Colombia.

Amorín da Silva, V.R. (2012). Contexto e impacto potencial del teleférico sobre la población de Complexo de Alemão, Rio de Janeiro. In J. D. Dávila (Ed.), Movilidad urbana y pobreza: aprendizajes de Medellín y Soacha, Colombia (pp. 186-193). Londres: Development Planning Unit, UCL, y Universidad Nacional de Colombia.

Bocarejo, J. P., Portilla, I. J., Velásquez, J. M., Cruz, M. N., Peña, A., \& Oviedo, D. R. (2014a). An innovative transit system and its impact on low income users: the case of the Metrocable in Medellín. Journal of Transport Geography, 39, 49-61. http://dx.doi.org/10.1016/j.jtrangeo.2014.06.018.

Bocarejo, J. P., Velásquez, J. M., \& Galarza, D. C. (2014b). Challenges of implementing à la mode transport projects: case studies of bus rapid transit and cable cars in Colombia. Transportation Research Record: Journal of the Transportation Research Board, 2451(1), 131-138. http://dx.doi.org/10.3141/2451-15.

Bogotá. Secretaría Distrital de Planeación. (2017a). Encuesta multipropósito 2017: principales resultados BogotáRegión. Bogotá. Recuperado el 10 de noviembre de 2019, de http://www.sdp.gov.co/sites/default/files/encuesta_multiproposito_2017__principales_resultados_bogota_region.pdf

Bogotá. Secretaría Distrital de Integración Social. (2017b). Localidad de Ciudad Bolívar: diagnóstico. Bogotá: Alcaldía Mayor de Bogotá D.C.

Bogotá. (2019). Conociendo mi localidad: Ciudad Bolívar es la localidad número 19 del Distrito Capital de Bogotá y se ubica en el suroccidente de la ciudad. Bogotá: Alcaldía Mayor de Bogotá D.C.. Recuperado el 10 de noviembre de 2019, de http://www.ciudadbolivar.gov.co/mi-localidad/conociendo-mi-localidad/historia" http://www.ciudadbolivar.gov.co/mi-localidad/conociendo-mi-localidad/historia

Brand, P., \& Dávila, J. (2011). Aerial cable-car systems for public transport in low-income urban areas: lessons from Medellin, Colombia. Recuperado el 1 de septiembre de 2019, de

http://opendocs.ids.ac.uk/opendocs/handle/123456789/11788 
Brand, P., \& Dávila, J. (2013). Metrocables and “Social Urbanism”: two complementary strategies. In J. D. Dávila (Ed.), Urban mobility and poverty: lessons from Medellín and Soacha, Colombia (pp. 46-54). London: UCL, UNC.

Carrión, F. (2013). El desafío politico del gobierno de la ciudad metropolitana en América. In C. Durán (Ed.), La gestión urbana en América Latina: debates desde la reflexión-acción (pp. 23-48). Bogotá: Universidad Piloto de Colombia.

Cervero, R. (2000). Informal transport in the developing world. Nairobi: UN-HABITAT.

Cervero, R., \& Golub, A. (2007). Informal transport: a global perspective. Transport Policy, 14(6), 445-457. http://dx.doi.org/10.1016/j.tranpol.2007.04.011.

Clichevsky, N. (2000). Informalidad y segregación urbana en América Latina: una aproximación (Serie Medio Ambiente y Desarrollo, No. 28). Santiago de Chile: CEPAL/Naciones Unidas. Recuperado el 1 de septiembre de 2019, de https://repositorio.cepal.org/bitstream/handle/11362/5712/S00100859_es.pdf?sequence=1\&isAllowed=y

Coupé, F. (2012). Los metrocables: riesgo, pobreza e inclusión. In J. D. Dávila (Ed.), Movilidad urbana y pobreza: aprendizajes de Medellín y Soacha, Colombia (pp. 57-69). Londres: Development Planning Unit, UCL, y Universidad Nacional de Colombia.

Coupé, F., \& Cardona, J. G. (2013). Impact of the metrocables on the local economy. In J. D. Dávila (Ed.), Urban mobility \& poverty: lessons from Medellín and Soacha, Colombia (pp. 89-103). London: Development Planning Unit, University College London.

Coupé, F., Brand, P., \& Dávila, J. (2013). Medellín: institutional context and urban paradigm change. In J. D. Dávila (Ed.), Urban mobility \& poverty: lessons from Medellín and Soacha, Colombia (pp. 55-67). London: UCL, UNC

Daste D. (2012). Iniciativas comunitarias y el tránsito al gobierno local: el MIOCABLE de Cali, Colombia. In J. D. Dávila (Ed.), Movilidad urbana y pobreza: aprendizajes de Medellín y Soacha, Colombia (pp. 158-169). Londres: Development Planning Unit, UCL, y Universidad Nacional de Colombia.

Dávila, J. D., \& Brand, P. C. (2012). La gobernanza del transporte público urbano: indagaciones alrededor de los Metrocables de Medellín. Bitácora Urbano-Territorial, 21(2), 17.

Dávila, J. D., \& Daste, D. (2012). Medellin's aerial cable-cars: social inclusion and reduced emissions. In UN Environment Programme - UNEP, UNEP-IPSRM cities, decoupling and urban infrastructure. México.

Derpic, J. C. (2018). Orden, seguridad y limpieza: el teleférico de La Paz y El Alto: diferenciación urbana, informalidad y Estado en Bolivia. In H. J. Suárez (Ed.), ¿Todo cambia? Reflexiones sobre el "proceso de cambio" en Bolivia (Cap. 1, p. 23). México: Instituto de Investigaciones Sociales, Universidad Nacional Autónoma de México. Recuperado el 1 de septiembre de 2019, de http://ru.iis.sociales.unam.mx/jspui/bitstream/IIS/5485/2/todo_cambia.pdf\#page=21

Dimitriou, H. T. (2013). Transport planning for third world cities. London: Routledge. http://dx.doi.org/10.4324/9780203768921.

Dureau, F., \& Gouëset, V. (2011). Formas de poblamiento y desigualdades en los desplazamientos. La evolución de la movilidad cotidiana en dos periferias populares de Bogotá: soacha y Madrid (1993-2009). Territorios, 2(25), 65-94.

Escobar, D. A., Tapasco, O. A., \& Giraldo, J. A. (2015). Medición de desempeño del sistema de transporte cable aéreo de la ciudad de manizales en Colombia, usando tres enfoques: analítico, simulado y de accesibilidad urbana. Información Tecnológica, 26(6), 199-210. http://dx.doi.org/10.4067/S0718-07642015000600020.

Espósito-Galarce, F., \& Silva, R. C. M. (2016). Apropiación, uso y abandono de los espacios residuales provocados por el teleférico del Complexo do Alemão, Río de Janeiro. Arquiteturarevista, 12(1), 11-23.

http://dx.doi.org/10.4013/arq.2016.121.02.

Gakenheimer, R. (1999). Urban mobility in the developing world. Transportation Research Part A, Policy and Practice, 33(7-8), 671-689. http://dx.doi.org/10.1016/S0965-8564(99)00005-1. 
Gakenheimer, R. (2012). Reflexiones sobre el uso del suelo y los Metrocables de Medellín. In J. D. Dávila (Ed.), Movilidad urbana y pobreza: aprendizajes de Medellín y Soacha, Colombia (pp. 114-117). Londres: Development Planning Unit, UCL, y Universidad Nacional de Colombia.

García, D. A. E., \& Orozco, F. J. G. (2011). Impacto de un sistema de transporte tipo Cable sobre la movilidad urbana. Caso Manizales (Colombia). Avances: Investigación en Ingeniería, 8(1), 92-98.

Garsous, G., Suárez-Alemán, A., \& Serebrisky, T. (2019). Cable cars in urban transport: travel time savings from La Paz-El Alto (Bolivia). Transport Policy, 75, 171-182. http://dx.doi.org/10.1016/j.tranpol.2017.05.005.

Golub, A., Balassiano, R., Araújo, A., \& Ferreira, E. (2009). Regulation of the informal transport sector in Rio de Janeiro, Brazil: welfare impacts and policy analysis. Transportation, 36(5), 601-616.

http://dx.doi.org/10.1007/s11116-009-9215-y.

Goodship, P. (2015). The impact of an urban cable car transport system on the spatial configuration of an informal settlement. In Proceedings of the 10th International Space Syntax Symposium. London: UCL. Recuperado el 1 de septiembre de 2019, de http://www.sss10.bartlett.ucl.ac.uk/wpcontent/uploads/2015/07/SSS10_Proceedings_131.pdf

Gwilliam, K. (2003). Urban transport in developing countries. Transport Reviews, 23(2), 197-216. http://dx.doi.org/10.1080/01441640309893.

Heinrichs, D., \& Bernet, J. S. (2014). Public transport and accessibility in informal settlements: aerial cable cars in Medellín, Colombia. Transportation Research Procedia, 4, 55-67. http://dx.doi.org/10.1016/j.trpro.2014.11.005.

Instituto de Desarrollo Urbano - IDU. (2019). Proyecto Transmicable Ciudad-Bolivar. Informe de satisfacción ciudadana Etapa Ex-post. Bogotá: Alcaldía de Bogotá.

Jaramillo, S. (2012). Urbanización informal: diagnósticos y políticas: una revisión al debate latinoamericano para pensar líneas de acción actuales (Serie Documentos CEDE, No. 009820). Bogotá: CEDE, Universidad de los Andes.

Jones, P., \& Lucas, K. (2012). Social impacts and equity issues in transport: an introduction. Journal of Transport Geography, 21, 1-3. http://dx.doi.org/10.1016/j.jtrangeo.2012.01.032.

Kaltheier, R. M. (2002). Urban transport and poverty in developing countries: analysis and options for transport policy and planning. Eschborn: GTZ.

Kowarick, L. (1996). Expoliación urbana, luchas sociales y ciudadanía: retazos de nuestra historia reciente. Estudios Sociológicos, 14(42), 729-743.

Lavadinho, S. (2014). Dinámicas de proximidad en la ciudad: ideas para la transformación urbana. Ciudades, (17), 21-39.

Leibler, L., \& Brand, P. (2012). Movilidad e inclusión social: la experiencia desde la periferia de Medellín y el primer Metrocable. Boletín del Instituto Francés de Estudios Andinos, 41(3), 363-387. http://dx.doi.org/10.4000/bifea.147.

Litman, T. (2007). Evaluating accessibility for transportation planning. Recuperado el 1 de septiembre de 2019, de https://trid.trb.org/view/1155070

Lomme, R. (2008). Should South African minibus taxis be scrapped? Formalizing informal urban transport in a developing country. In Proceedings of the CODATU XIII Conference. Paris: EMTA.

Martínez, J. L. (2019). TransMiCable ha transportado a 1.8 millones de pasajeros. Bogotá. Recuperado el 10 de diciembre de 2019, de https://bogota.gov.co/mi-ciudad/movilidad/pasajeros-de-transmicable

Metro de Medellín. (2012). Innovación, la apuesta por la movilidad sostenible. Revista METRO, 3, 6-10. Recuperado el 19 de junio de 2019, de https://www.metrodemedellin.gov.co/Portals/1/Images/Contenido/REVISTASOTROS/2011_mag_metrocable.pdf

Milan, B. F., \& Creutzig, F. (2017). Lifting peripheral fortunes: upgrading transit improves spatial, income and gender equity in Medellin. Cities, 70, 122-134. http://dx.doi.org/10.1016/j.cities.2017.07.019. 
Miralles-Guasch, C., \& Sardà, O. M. (2013). Dinámicas de proximidad en ciudades multifuncionales. Ciudad y Territorio Estudios Territoriales, 45(177), 501-510.

Mitlin, D., \& Satterthwaite, D. (2013). Urban poverty in the global south: scale and nature. New York: Routledge.

Moreno Luna, C., \& Rubiano Bríñez, M. (2014). Segregación residencial y movilidad cotidiana en el contexto metropolitano: un estudio a partir de las relaciones Bogotá - Soacha. Territorios, 16(31), 133-162. http://dx.doi.org/10.12804/territ31.2014.06.

Naranjo, N. (2012). Metrocable de San Agustí, Caracas: el precio de la integración a la ciudad. In J. D. Dávila (Ed.), Movilidad urbana y pobreza: aprendizajes de Medellín y Soacha, Colombia (pp. 158-169). Londres: Development Planning Unit, UCL, y Universidad Nacional de Colombia.

Oviedo-Hernández, D., \& Titheridge, H. (2016). Mobilities of the periphery: Informality, access and social exclusion in the urban fringe in Colombia. Journal of Transport Geography, 55, 152-164.

http://dx.doi.org/10.1016/j.jtrangeo.2015.12.004.

Páramo, P. (2011). Investigación de estudio de caso: estrategia de indagación. In P. Páramo (Ed.), La investigación en ciencias sociales: estrategias de investigación. Bogotá: Compilador Pablo Páramo.

Pírez, P. (2013). La urbanización y la política de los servicios urbanos en América Latina. Andamios, 10(22), 45-67. http://dx.doi.org/10.29092/uacm.v10i22.266.

Sarmiento, I., Córdoba, J., Mejía, Á. G., \& Agudelo, L. (2013). Metrocables and travel patterns in medellín: the inclusion of latent variables in transport models. In J. D. Dávila (Ed.), Urban mobility and poverty: lessons from Medellín and Soacha, Colombia (pp. 81-88). London: UCL, UNC.

Sengers, F., \& Raven, R. (2014). Metering motorbike mobility: informal transport in transition? Technology Analysis and Strategic Management, 26(4), 453-468. http://dx.doi.org/10.1080/09537325.2013.870991.

Shimazaki, T., \& Rahman, M. (1996). Physical characteristics of paratransit in developing countries of Asia. Journal of Advanced Transportation, 30(2), 5-24. http://dx.doi.org/10.1002/atr.5670300203.

Transmilenio. (2019, 11 de abril). Balance de operación del primer trimestre de TransMiCable. Recuperado el 19 de diciembre de 2019, de https://www.transmilenio.gov.co/publicaciones/151190/balance-de-operacion-delprimer-trimestre-de-transmicable/

Ureta, S. (2008). To move or not to move? Social exclusion, accessibility and daily mobility among the low-income population in Santiago, Chile. Mobilities, 3(2), 269-289. http://dx.doi.org/10.1080/17450100802095338.

Vasconcellos, E. A. (2014). Urban transport environment and equity: the case for developing countries. Oxon: Earthscan. http://dx.doi.org/10.4324/9781315071756.

Veeduría Distrital. (2019). Ficha UPZ: Lucero - Ciudad Bolívar. Recuperado el 10 de junio de 2019, de http://veeduriadistrital.gov.co/sites/default/files/files/UPZ/LUCERO.pdf

Villar-Uribe, J. R. (2017). El rol del transporte informal en Santiago de Cali. ¿Problema u oportunidad? Papeles de Coyuntura, 43, 40-77. Recuperado el 10 de junio de 2019, de www.papelesdecoyuntura.com

Editor: Fábio Duarte

Recibido: Dic. 13, 2019

Aprobado: Sept. 04, 2020 